\title{
Color superconductivity and the strange quark
}

\author{
Mark Alford \\ Physics Department \\ Washington University \\ Saint Louis, MO 63130 \\ USA
}

Nov 22, 2005

\begin{abstract}
At ultra-high density, matter is expected to form a degenerate Fermi gas of quarks in which there is a condensate of Cooper pairs of quarks near the Fermi surface: color superconductivity. In these proceedings I review some of the underlying physics, and discuss outstanding questions about the phase structure of ultra-dense quark matter.
\end{abstract}




\section{Introduction}

The exploration of the phase diagram of matter at ultra-high temperature or density is an area of great interest and activity, both on the experimental and theoretical fronts. Heavyion colliders such as the SPS at CERN and RHIC at Brookhaven have probed the hightemperature region, searching for the transition to deconfined quark matter. In this paper we discuss a different part of the phase diagram, the low-temperature high-density region. Here there are as yet no experimental constraints, but we expect to find phases characterized by Cooper pairing of quarks, i.e. color superconductivity, driven by the Bardeen-CooperSchrieffer (BCS) 1] mechanism. The BCS mechanism operates when there exists an attractive interaction between fermions at a Fermi surface. The QCD quark-quark interaction is strong, and is attractive in many channels, so we expect cold dense quark matter to generically exhibit color superconductivity. Moreover, quarks, unlike electrons, have color and flavor as well as spin degrees of freedom, so many different patterns of pairing are possible. This leads us to expect a rich phase structure in matter beyond nuclear density.

Calculations using a variety of methods agree that at sufficiently high density, the favored phase is color-flavor-locked (CFL) color-superconducting quark matter [2] (for reviews, see Ref. [3]). However, there is still uncertainty over the nature of the next phase down in density. Recent work [4 suggests that when the density drops low enough so that the mass of the strange quark can no longer be neglected, there is a continuous phase transition from the CFL phase to a new gapless CFL (gCFL) phase, which could lead to observable consequences if it occurred in the cores of neutron stars [5]. However, it now appears that some of the gluons in the gCFL phase have imaginary Meissner masses, indicating an instability towards an unknown lower-energy phase [6, 17, 8, 9]. The nature of this phase is still unclear, although the crystalline "LOFF" phase is a strong candidate [10, 8].

\section{Review of color superconductivity}

\subsection{The phase diagram of quark matter}

In the real world there are two light quark flavors, the up $(u)$ and down $(d)$, with masses $\lesssim 5 \mathrm{MeV}$, and a medium-weight flavor, the strange $(s)$ quark, with mass $\sim 100 \mathrm{MeV}$. (Their effective "constituent" masses in dense matter may be much larger.) The strange quark therefore plays a crucial role in the phases of QCD. Fig. 1 shows a conjectured phase diagram for QCD, and also a calculated phase diagram obtained using a Nambu-Jona-Lasinio model of QCD. In both cases, along the horizontal axis the temperature is zero, and the density rises from the onset of nuclear matter through the transition to quark matter. Compact stars are in this region of the phase diagram, although it is not known whether their cores are dense enough to reach the quark matter phase. Along the vertical axis the temperature rises, taking us through the crossover from a hadronic gas to the quark gluon plasma. This is the regime explored by high-energy heavy-ion colliders.

At the highest densities we find the CFL phase, in which the strange quark participates symmetrically with the up and down quarks in Cooper pairing - this is described in more detail below. The phases that occur at intermediate density are still not well understood. The NJL calculation works with a limited set of possibilities, and the NJL calculation neglects more 
$\underline{\text { Conjectured form }}$

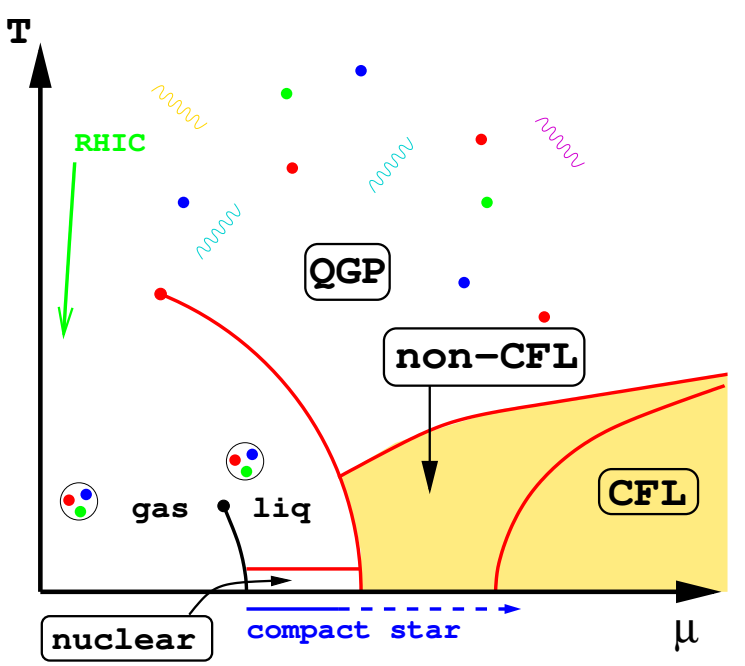

$\underline{\text { NJL calculation }}$

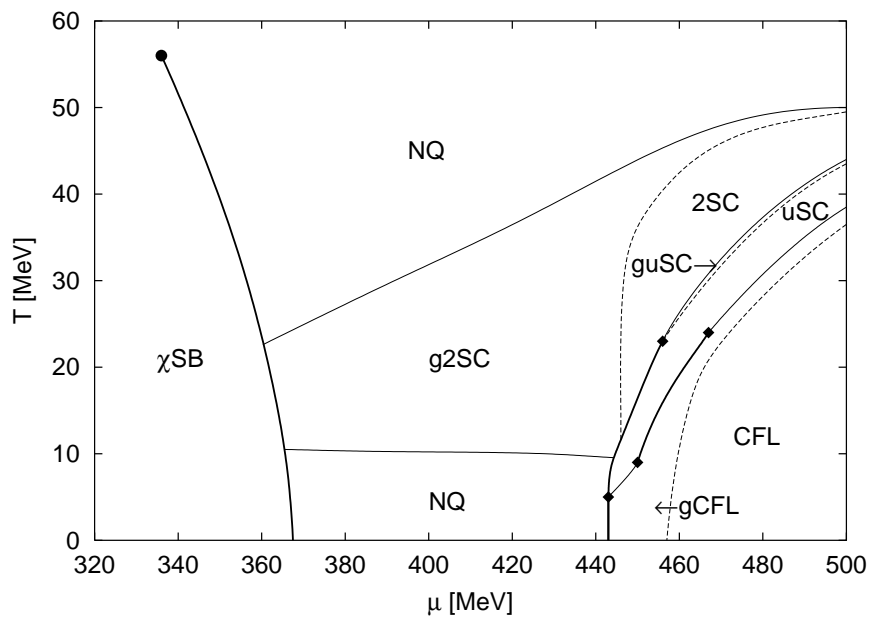

Figure 1: On the left, the conjectured form of the phase diagram for matter at ultra-high density and temperature. On the right, the result of a calculation using a NJL model [17]. At high density we find a rich structure of color-superconducting phases.

exotic possibilities such as kaon condensation [11, crystalline color superconductivity (LOFF) [10], and single-flavor pairing [12, 13, 14, 15, 16].

\subsection{Color superconductivity}

The fact that QCD is asymptotically free implies that at sufficiently high density and low temperature, there is a Fermi surface of weakly-interacting quarks. The interaction between these quarks is certainly attractive in some channels (quarks bind together to form baryons), so we expect the formation of a condensate of Cooper pairs. We can see this by considering the grand canonical potential $F=E-\mu N$, where $E$ is the total energy of the system, $\mu$ is the chemical potential, and $N$ is the number of quarks. The Fermi surface is defined by a Fermi energy $E_{F}=\mu$, at which the free energy is minimized, so adding or subtracting a single particle costs zero free energy. Now switch on a weak attractive interaction. It costs no free energy to add a pair of particles (or holes), and if they have the right quantum numbers then the attractive interaction between them will lower the free energy of the system. Many such pairs will therefore be created in the modes near the Fermi surface, and these pairs, being bosonic, will form a condensate. The ground state will be a superposition of states with all numbers of pairs, breaking the fermion number symmetry.

A pair of quarks cannot be a color singlet, so the resulting condensate will break the local color symmetry $S U(3)_{\text {color. }}$. The formation of a condensate of Cooper pairs of quarks is therefore called "color superconductivity". The condensate plays the same role here as the Higgs particle does in the standard model: the color-superconducting phase can be thought of as the Higgs phase of QCD. 


\subsection{Highest density: Color-flavor locking (CFL)}

At the highest densities, where the strange quark Fermi momentum is close to the up and down quark Fermi momenta, the favored phase is "color-flavor locking" (CFL) 2]. This has been confirmed by both NJL [2, 18] and gluon-mediated interaction calculations [19]. The CFL pairing pattern is

$$
\begin{aligned}
\left\langle q_{i}^{\alpha} C \gamma_{5} q_{j}^{\beta}\right\rangle_{1 P I} & \propto(\kappa+1) \delta_{i}^{\alpha} \delta_{j}^{\beta}+(\kappa-1) \delta_{j}^{\alpha} \delta_{i}^{\beta}=\epsilon^{\alpha \beta N} \epsilon i j N+\kappa(\cdots) \\
{\left[S U(3)_{\text {color }}\right] } & \times \underbrace{S U(3)_{L} \times S U(3)_{R}}_{\supset\left[U(1)_{Q}\right]} \times U(1)_{B} \rightarrow \underbrace{S U(3)_{C+L+R}}_{\supset\left[U(1)_{\tilde{Q}}\right]} \times \mathbb{Z}_{2}
\end{aligned}
$$

Color indices $\alpha, \beta$ and flavor indices $i, j$ run from 1 to 3 , Dirac indices are suppressed, and $C$ is the Dirac charge-conjugation matrix. The term multiplied by $\kappa$ corresponds to pairing in the $\left(\mathbf{6}_{S}, \mathbf{6}_{S}\right)$, which although not energetically favored breaks no additional symmetries and so $\kappa$ is in general small but not zero [2, 19, 20, 21]. The Kronecker deltas connect color indices with flavor indices, so that the condensate is not invariant under color rotations, nor under flavor rotations, but only under simultaneous, equal and opposite, color and flavor rotations. Since color is only a vector symmetry, this condensate is only invariant under vector flavor+color rotations, and breaks chiral symmetry. The features of the CFL pattern of condensation are

- The color gauge group is completely broken. All eight gluons become massive. This ensures that there are no infrared divergences associated with gluon propagators.

- All the quark modes are gapped. The nine quasiquarks (three colors times three flavors) fall into an $\mathbf{8} \oplus \mathbf{1}$ of the unbroken global $S U(3)$, so there are two gap parameters. The singlet has a larger gap than the octet.

- A rotated electromagnetism (" $\tilde{Q} ")$ survives unbroken. It is a combination of the original photon and one of the gluons.

- Two global symmetries are broken, the chiral symmetry and baryon number, so there are two gauge-invariant order parameters that distinguish the CFL phase from the QGP, and corresponding Goldstone bosons which are long-wavelength disturbances of the order parameter. When the light quark mass is non-zero it explicitly breaks the chiral symmetry and gives a mass to the chiral Goldstone octet, but the CFL phase is still a superfluid, distinguished by its baryon number breaking.

- The symmetries of the 3-flavor CFL phase are the same as those one might expect for 3-flavor hypernuclear matter [18, so it is possible that there is no phase transition between them.

\section{Real-world quark matter}

\subsection{Stresses on the CFL phase}

The CFL phase is characterized by pairing between different flavors and different colors of quarks. We can easily understand why this is to be expected. Firstly, the QCD interaction between two quarks is most attractive in the channel that is antisymmetric in color (the $\overline{\mathbf{3}}$ ). Secondly, pairing tends to be stronger in channels that do not break rotational symmetry 
[12, 13, 14, 15, 16], so we expect the pairing to be a spin singlet, i.e. antisymmetric in spin. Finally, fermionic antisymmetry of the Cooper pair wavefunction then forces the Cooper pair to be antisymmetric in flavor.

Pairing between different colors/flavors can occur easily when they all have the same chemical potentials and Fermi momenta. This is the situation at very high density, where the strange quark mass is negligible. However, in a real compact star we must take into account the forces that try to split those Fermi momenta apart, imposing an energy cost on crossspecies pairing. We must require electromagnetic and color neutrality [22, 23] (possibly via mixing of oppositely-charged phases), allow for equilibration under the weak interaction, and include a realistic mass for the strange quark. These factors cause the different colors and flavors to have different chemical potentials, and this imposes a stress on cross-species pairing such as occurs in the CFL pairing pattern. As we come down in density, we expect the CFL pairing pattern to be distorted, and then to be replaced by some other pattern.

In the next few subsections we give a quick overview of the expected phases of real-world quark matter. We restrict our discussion to zero temperature because the critical temperatures for most of the phases that we discuss are expected to be of order $10 \mathrm{MeV}$ or higher, and the core temperature of a neutron star is believed to drop below this value within minutes (if not seconds) of its creation in a supernova.

\subsection{Kaon condensation: the CFL- $K^{0}$ phase}

Bedaque and Schäfer [11] showed that when the stress is not too large (high density), it may simply modify the CFL pairing pattern by inducing a flavor rotation of the condensate which can be interpreted as a condensate of " $K^{0}$ " mesons, i.e. the neutral anti-strange Goldstone bosons associated with the chiral symmetry breaking. This is the "CFL-K0" phase, which breaks isospin. The $K^{0}$ condensate can easily be suppressed by instanton effects [24], but if these are ignored then the kaon condensation occurs for $M_{s} \gtrsim m^{1 / 3} \Delta^{2 / 3}$ for light $(u$ and $d$ ) quarks of mass $m$. This was demonstrated using an effective theory of the Goldstone bosons, but with some effort can also be seen in an NJL calculation [25, 26].

\subsection{The non-CFL region}

The nature of the next significant transition has been studied in NJL model calculations which ignore the $K 0$-condensation in the CFL phase [27, 28, 17]. It has been found that the phase structure depends on the strength of the pairing. If the pairing is very strong (so that $\Delta_{C F L} \sim 100 \mathrm{MeV}$ where $\Delta_{C F L}$ is what the CFL gap would be at $\mu \sim 500 \mathrm{MeV}$ if $M_{s}$ were zero) then the CFL phase survives all the way down to the transition to nuclear matter. For less strong pairing, there may be a transition to a two-flavor pairing ("2SC") phase [29, 30] and/or a single-flavor pairing phase (see below), and then to nuclear matter.

For a wide range of parameter values, however, we find something more interesting. We can

make a rough quantitative analysis by expanding in powers of $M_{s} / \mu$ and $\Delta / \mu$, and ignoring the fact that the effective strange quark mass may be different in different phases [23. Such an analysis shows that as we come down in density we find a transition at $\mu \approx \frac{1}{2} M_{s}^{2} / \Delta_{C F L}$ from CFL to another phase, the gapless CFL phase (gCFL) 4]. The gCFL phase is also found in more complete NJL calcualtions that do not use the assumptions of Ref 4]. This is seen in the right-hand panel of Fig. 1 (the "gCFL" region) and is discussed in detail below. 


\subsection{Single-flavor pairing}

If $M_{s}$ is sufficiently large at densities where quark matter is favored over nuclear matter, then (via the neutrality requirement) it splits the chemical potentials of the different flavors so far apart that no cross-species pairing can occur at all. There is no CFL or 2SC pairing. In most NJL studies this is described loosely as "unpaired" quark matter. However, it is well known that there are attractive channels for a single flavor, although they are much weaker than the 2SC and CFL channels. Thus in these regions we expect some form of single-flavor pairing. There are various "1SC" phases, many of which break rotational invariance, and a very interesting color-spin-locked (CSL) phase which is rotationally invariant [12, 13, 14, 15, 16. These phases have much lower critical temperatures than the others (from a few $\mathrm{MeV}$ down to $\mathrm{eV}$ ).

\subsection{The gapless CFL phase}

As mentioned above, an expansion in $M_{s} / \mu$ for pairing strength $\Delta_{C F L} \lesssim 25 \mathrm{MeV}$ shows that, for $M_{s}^{2} / \mu \gtrsim 2 \Delta$, the CFL phase has higher free energy than an alternative phase called gapless CFL ("gCFL"). This follows from the energetic balance, mentioned above, between the cost of keeping the Fermi surfaces together and the benefit of the pairing that can then occur. The leading effect of $M_{s}$ is like a shift in the chemical potential of the strange quarks, so the $b d$ and gs quarks feel "effective chemical potentials" $\mu_{b d}^{\text {eff }}=\mu-\frac{2}{3} \mu_{8}$ and $\mu_{g s}^{\text {eff }}=\mu+\frac{1}{3} \mu_{8}-\frac{M_{s}^{2}}{2 \mu}$. In the CFL phase $\mu_{8}=-M_{s}^{2} /(2 \mu)$ [23], so $\mu_{b d}^{\text {eff }}-\mu_{g s}^{\text {eff }}=M_{s}^{2} / \mu$. The CFL phase will be stable as long as the pairing makes it energetically favorable to maintain equality of the $b d$ and $g s$ Fermi momenta, despite their differing chemical potentials 31. It becomes unstable when the energy gained from turning a gs quark near the common Fermi momentum into a bd quark (namely $M_{s}^{2} / \mu$ ) exceeds the cost in lost pairing energy $2 \Delta_{1}$. So the CFL phase is stable when

$$
\frac{M_{s}^{2}}{\mu}<2 \Delta_{C F L},
$$

For larger $M_{s}^{2} / \mu$, the CFL phase is replaced by some new phase with unpaired $b d$ quarks, which cannot be neutral unpaired or $2 \mathrm{SC}$ quark matter because the new phase and the CFL phase must have the same free energy at the critical $M_{s}^{2} / \mu=2 \Delta_{C F L}$.

The obvious approach to finding this phase is to perform a NJL model calculation with a general ansatz for the pairing that includes differences between the flavors, for example by allowing different pairing strengths $\Delta_{u d}, \Delta_{d s}, \Delta_{u s}$. This was done in Ref. [4], and the resultant "gCFL" phase was described in detail. In Fig 2 we show the results. The gCFL phase takes

over from CFL at $M_{s}^{2} / \mu \approx 2 \Delta_{C F L}$, and remains favored beyond the value $M_{s}^{2} / \mu \approx 4 \Delta_{C F L}$ at which the CFL phase would become unfavored.

\subsection{Crystalline pairing}

The pairing patterns discussed so far have been translationally invariant. But in the region of parameter space where cross-species pairing is just barely excluded by stresses that pull apart the Fermi surfaces, one expects a position-dependent pairing known as the "LOFF" phase [33, 10, 34, 35. This arises because one way to achieve pairing between different flavors while accomodating the tendency for the Fermi momenta to separate is to only pair over part of 


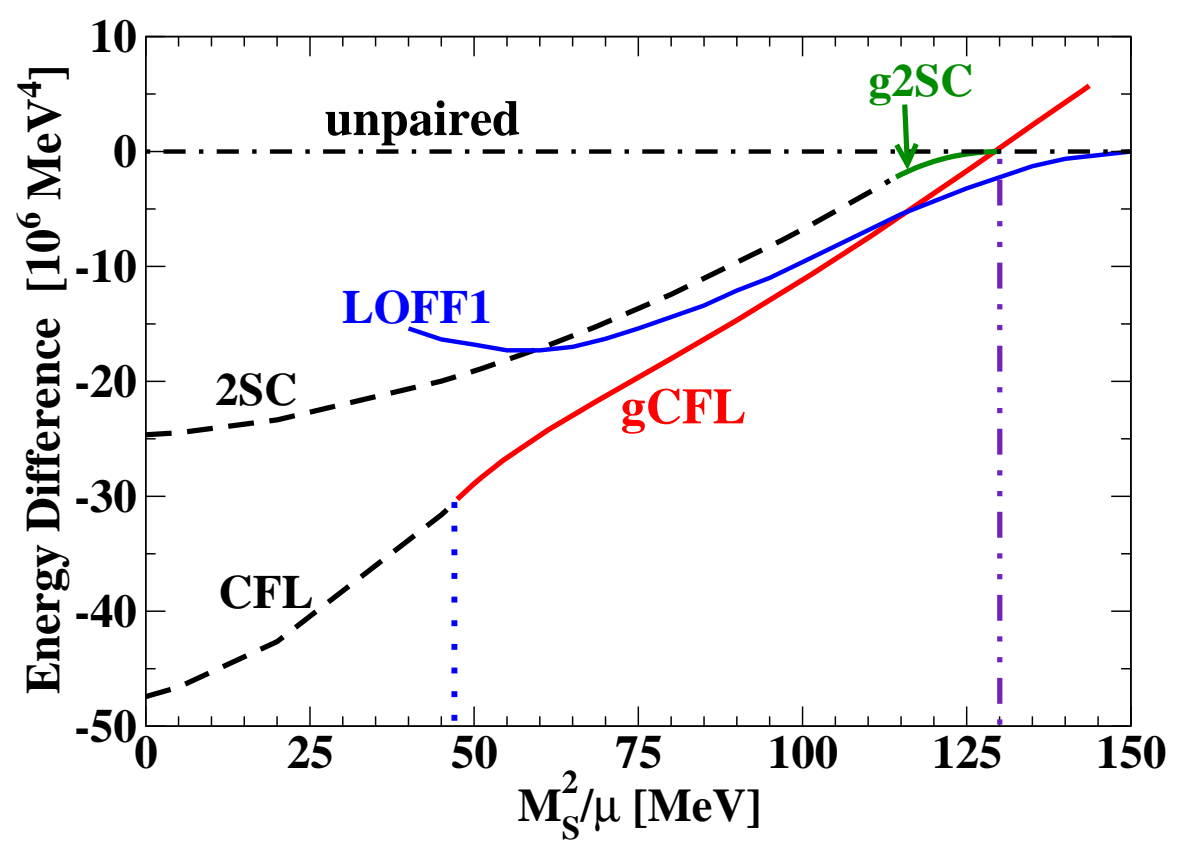

Figure 2: Free energy of various phases in an NJL model, allowing different pairing strengths $\Delta_{u d}, \Delta_{d s}, \Delta_{u s}$ for the different flavors. The CFL pairing strength is $\Delta_{C F L}=25 \mathrm{MeV}$. Note that the gCFL phase takes over from CFL at $M_{s}^{2} / m u \approx 2 \Delta_{C F L}$, and remains favored beyond the value $M_{s}^{2} / \mu \approx 4 \Delta_{C F L}$ at which the CFL phase would become unfavored. The "LOFF1" curve is the single-plane-wave LOFF ansatz of [32].

the Fermi surface. As we will discuss below, the LOFF phase competes with the gCFL phase, and may resolve that phase's stability problems.

\subsection{Mixed Phases}

Another way for a system to deal with a stress on its pairing pattern is phase separation. In the context of quark matter this corresponds to relaxing the requirement of local charge neutrality, and requiring neutrality only over long distances, so we allow a mixture of a positively charged and a negatively charged phase, with a common pressure and a common value of the electron chemical potential $\mu_{e}$ that is not equal to the neutrality value for either phase. Such a mixture of nuclear and CFL quark matter was studied in Ref. [36]. In quark matter it has been found that as long as we require local color neutrality such mixed phases are not the favored response to the stress imposed by the strange quark mass [4, 37. Phases involving color charge separation have been studied [38] but it seems likely that the energy cost of the color-electric fields will disfavor them.

\subsection{Beyond gapless CFL}

The arguments above led us to the conclusion that the favored phase of quark matter at the highest densities is the CFL phase, and that as the density is decreased there is a transition to another color superconducting phase, the gapless CFL phase. However, it turns out that the gCFL phase is itself unstable, and that there is therefore another phase of even lower free 


\section{Gapless CFL phase}

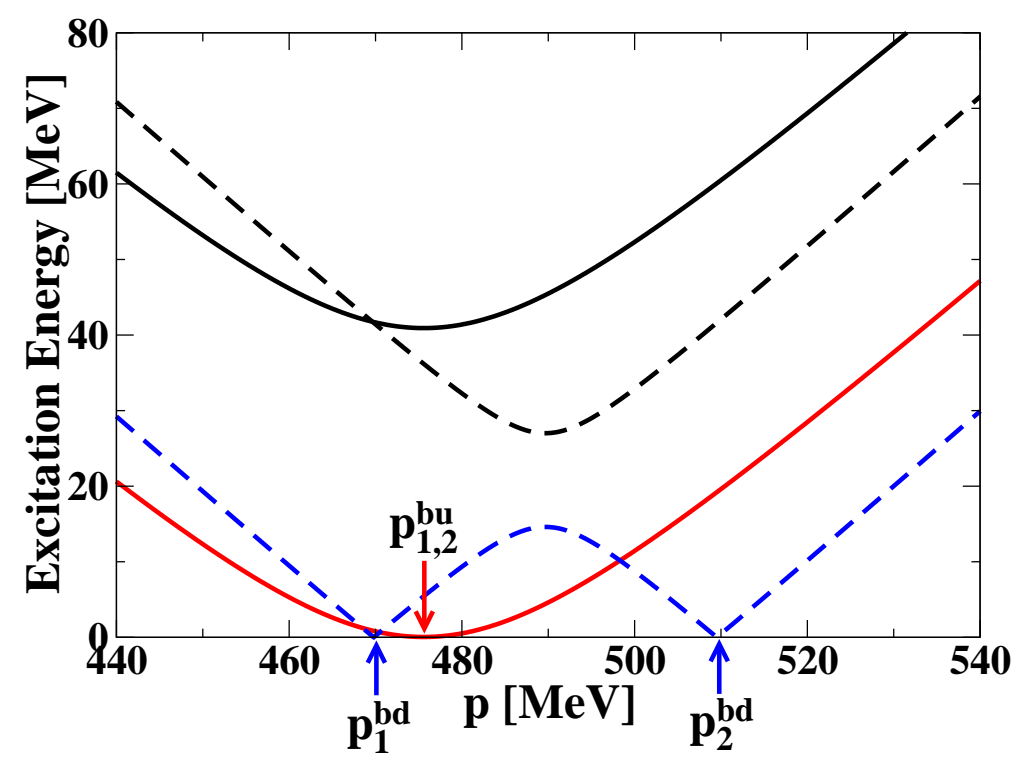

Figure 3: Dispersion relations of the lightest quasiquark excitations in the gCFL phase, at $\mu=500 \mathrm{MeV}$, with $m_{s}=200 \mathrm{MeV}$ and $\Delta_{C F L}=25 \mathrm{MeV}$. Note that in there is a gapless mode with a quadratic dispersion relation (energy reaching zero at momentum $p_{1,2}^{b u}$ ) as well as two gapless modes with more conventional linear dispersion relations.

energy, that occurs below the gCFL phase in the phase diagram. The nature of that phase remains uncertain at present.

The instability of the gCFL phase appears to be related to one of its most interesting features, namely the presence of gapless fermionic excitations around the ground state. These are illustrated in Fig. 3, which shows that there is one mode (the bu-rs quasiparticle) with an unusual quadratic dispersion relation, which is expected to give rise to exotic transport properties [5]. The instability of the gCFL phase was established in Refs. 7, 9] after an analogous instability in the gapless 2SC phase had been discovered [6, 8]. The instability manifests itself in imaginary Meissner masses $M_{M}$ for some of the gluons. $M_{M}^{2}$ is the low-momentum current-current two-point function, and $M_{M}^{2} /\left(e^{2} \Delta^{2}\right)$ is the coefficient of the gradient term in the effective theory of small fluctuations around the ground-state condensate. The fact that we find a negative value when the quasiparticles are gapless indicates an instability towards spontaneous breaking of translational invariance. Calculations in a simple two-species model [39] show that imaginary $M_{M}$ is generically associated with the presence of gapless charged fermionic modes.

The nature of the true ground state in the intermediate density regime remains unclear. It could be a mixed phase [40], a crystalline (LOFF) phase [8], or a $p$-wave meson condensate 41, 42. Recent calculations [32] for the 3-flavor case show that even a very simple LOFF ansatz yields a state that has lower free energy than gCFL in the region where the gCFL $\rightarrow$ unpaired transition occurs (see Fig. 21). Based on what was found in the two-flavor case [34, it is reasonable to expect that when the full space of crystal structures is explored, the LOFF state will be preferred to gCFL over a much wider range of the stress parameter $M_{s}^{2} \Delta /(2 \mu)$, 
and it might turn out that the whole gCFL region is actually a LOFF region.

An alternative explanation was advanced by Hong [43] (see also Ref. [44]): since the instability is generically associated with the presence of gapless fermionic modes, and the BCS mechanism implies that any gapless fermionic mode is unstable to Cooper pairing in the most attractive channel, one might expect that the instability will simply be resolved by "secondary pairing". This means the formation of a $\langle q q\rangle$ condensate where $q$ is either one of the gapless quasiparticles whose dispersion relation is shown in Fig. 3. After the formation of such a secondary condensate, the linear gapless dispersion relations would be modified by "rounding out" of the corner where the energy falls to zero, leaving a secondary energy gap $\Delta_{s}$, which renders the mode gapped, and removes the instability. In the case of the quadratically gapless mode there is a greatly increased density of states at low energy (in fact, the density of states diverges as $E^{-1 / 2}$ ), so Hong calculated that the secondary pairing should be much stronger than would be predicted by BCS theory, and he specifically predicted $\Delta_{s} \propto G_{s}^{2}$ for coupling strength $G_{s}$, as compared with the standard BCS result $\Delta \propto \exp (-$ const $/ G)$.

This possibility was worked out in an NJL model in Ref. [45], using a two-species model. This allowed a detailed exploration of the strength of secondary pairing. The calculation confirmed Hong's prediction that in typical secondary channels $\Delta_{s} \propto G_{s}^{2}$. However, in all the secondary channels that were analyzed it was found that the secondary gap, even with this enhancement, is from ten to hundreds of times smaller than the primary gap at reasonable values of the secondary coupling. This shows that that secondary pairing does not generically resolve the magnetic instability of the gapless phase, since it indicates that there is a temperature range $\Delta_{s} \ll T \ll \Delta_{p}$ in which there is primary pairing (of strength $\Delta_{p}$ ) but no secondary pairing, and at those temperatures the instability problem would arise again.

\section{Conclusion}

As I have described, the project of delineating a plausible phase diagram for high-density quark matter is still not complete. I have discussed some ideas for the "non-CFL" region, but there are others such as a suggested gluon condensation in two-flavor quark matter [46], and deformation of the Fermi surfaces (discussed so far only in non-beta-equilibrated nuclear matter [47]). It is very interesting to note that the problem of how a system with pairing responds to a stress that separates the chemical potentials of the pairing species is a very generic one, arising in condensed matter systems and cold atom systems as well as in quark matter. Recent work by Son and Stephanov [48] on a two-species model characterized by a diluteness parameter and a splitting potential shows that between the BCS-paired region and the unpaired region in the phase diagram one should expect a translationally-broken region. In QCD this could correspond to a $p$-wave meson condensate or a LOFF state (see above). What is particularly exciting is that the technology of cold atom traps has advanced to the point where fermion superfluidity can now be seen in conditions where many of the important parameters can be manipulated, and it may soon be possible to investigate the response of the pairing to external stress under controlled experimental conditions. 


\section{References}

[1] J. Bardeen, L. Cooper, J. Schrieffer, Phys. Rev. 106, 162 (1957); Phys. Rev. 108, 1175 (1957)

[2] M. Alford, K. Rajagopal and F. Wilczek, Nucl. Phys. B537, 443 (1999) hep-ph/9804403.

[3] K. Rajagopal and F. Wilczek, hep-ph/0011333 M. G. Alford, Ann. Rev. Nucl. Part. Sci. 51 (2001) 131 hep-ph/0102047. D. K. Hong, Acta Phys. Polon. B 32, 1253 (2001) hep-ph/0101025|. D. H. Rischke, Prog. Part. Nucl. Phys. 52, 197 (2004) nucl-th/0305030|. T. Schäfer, hep-ph/0304281. S. Reddy, Acta Phys. Polon. B 33, 4101 (2002) arXiv:nucl-th/0211045.

[4] M. Alford, C. Kouvaris and K. Rajagopal, Phys. Rev. Lett. 92, 222001 (2004) arXiv:hep-ph/0311286; M. Alford, C. Kouvaris and K. Rajagopal, arXiv:hep-ph/0406137.

[5] M. Alford, P. Jotwani, C. Kouvaris, J. Kundu and K. Rajagopal, arXiv:astro-ph/0411560.

[6] M. Huang and I. A. Shovkovy, Phys. Rev. D 70, 094030 (2004) arXiv:hep-ph/0408268.

[7] R. Casalbuoni, R. Gatto, M. Mannarelli, G. Nardulli and M. Ruggieri, arXiv:hep-ph/0410401

[8] I. Giannakis and H. C. Ren, arXiv:hep-ph/0412015.

[9] K. Fukushima, arXiv:hep-ph/0506080.

[10] M. G. Alford, J. A. Bowers and K. Rajagopal, Phys. Rev. D 63, 074016 (2001) arXiv:hep-ph/0008208.

[11] P. F. Bedaque and T. Schäfer, Nucl. Phys. A 697 (2002) 802 hep-ph/0105150.

[12] M. Iwasaki, T. Iwado, Phys. Lett. B350, 163 (1995); M. Iwasaki, Prog. Theor. Phys. Suppl. 120, 187 (1995)

[13] T. Schafer, Phys. Rev. D 62, 094007 (2000) arXiv:hep-ph/0006034.

[14] M. Buballa, J. Hosek and M. Oertel, Phys. Rev. Lett. 90, 182002 (2003) arXiv:hep-ph/0204275.

[15] M. G. Alford, J. A. Bowers, J. M. Cheyne and G. A. Cowan, Phys. Rev. D 67, 054018 (2003) arXiv:hep-ph/0210106.

[16] A. Schmitt, Q. Wang and D. H. Rischke, Phys. Rev. D 66, 114010 (2002) arXiv:nucl-th/0209050.

[17] S. B. Ruster, V. Werth, M. Buballa, I. A. Shovkovy and D. H. Rischke, Phys. Rev. D 72, 034004 (2005) arXiv:hep-ph/0503184.

[18] T. Schafer and F. Wilczek, Phys. Rev. D 60, 074014 (1999) arXiv:hep-ph/9903503. 
[19] T. Schafer, Nucl. Phys. B 575, 269 (2000) arXiv:hep-ph/9909574.

[20] I. A. Shovkovy and L. C. R. Wijewardhana, Phys. Lett. B 470, 189 (1999) arXiv:hep-ph/9910225.

[21] R. D. Pisarski and D. H. Rischke, "Why color-flavor locking is just like chiral symmetry breaking". To be published in, Proceedings of the Judah Eisenberg Memorial Symposium, "Nuclear Matter, Hot and Cold", Tel Aviv, April 14 - 16, 1999 nucl-th/9907094.

[22] K. Iida and G. Baym, Phys. Rev. D 63, 074018 (2001) [Erratum-ibid. D 66, 059903 (2002)] arXiv:hep-ph/0011229.

[23] M. Alford and K. Rajagopal, JHEP 0206, 031 (2002) arXiv:hep-ph/0204001.

[24] T. Schafer, Phys. Rev. D 65, 094033 (2002) arXiv:hep-ph/0201189.

[25] M. Buballa, Phys. Lett. B 609, 57 (2005) arXiv:hep-ph/0410397.

[26] M. M. Forbes, arXiv:hep-ph/0411001.

[27] K. Fukushima, C. Kouvaris and K. Rajagopal, Phys. Rev. D 71, 034002 (2005) arXiv:hep-ph/0408322.

[28] D. Blaschke, S. Fredriksson, H. Grigorian, A. M. Oztas and F. Sandin, arXiv:hep-ph/0503194.

[29] M. Alford, K. Rajagopal and F. Wilczek, Phys. Lett. B422, 247 (1998) hep-ph/9711395.

[30] R. Rapp, T. Schäfer, E. V. Shuryak and M. Velkovsky, Phys. Rev. Lett. 81, 53 (1998) hep-ph/9711396.

[31] K. Rajagopal and F. Wilczek, Phys. Rev. Lett. 86, 3492 (2001) hep-ph/0012039.

[32] R. Casalbuoni, R. Gatto, N. Ippolito, G. Nardulli and M. Ruggieri, arXiv:hep-ph/0507247.

[33] A. I. Larkin and Yu. N. Ovchinnikov, Zh. Eksp. Teor. Fiz. 47, 1136 (1964) [Sov. Phys. JETP 20, 762 (1965)]; P. Fulde and R. A. Ferrell, Phys. Rev. 135, A550 (1964).

[34] J. A. Bowers and K. Rajagopal, Phys. Rev. D 66, 065002 (2002) arXiv:hep-ph/0204079.

[35] R. Casalbuoni and G. Nardulli, Rev. Mod. Phys. 76, 263 (2004) arXiv:hep-ph/0305069.

[36] M. G. Alford, K. Rajagopal, S. Reddy and F. Wilczek, Phys. Rev. D 64, 074017 (2001) arXiv:hep-ph/0105009.

[37] M. Alford, C. Kouvaris and K. Rajagopal, arXiv:hep-ph/0407257.

[38] F. Neumann, M. Buballa and M. Oertel, Nucl. Phys. A 714, 481 (2003) arXiv:hep-ph/0210078.

[39] M. Alford and Q. h. Wang, J. Phys. G 31, 719 (2005) arXiv:hep-ph/0501078. 
[40] S. Reddy and G. Rupak, Phys. Rev. C 71, 025201 (2005) arXiv:nucl-th/0405054.

[41] T. Schafer, arXiv:hep-ph/0508190.

[42] A. Kryjevski, arXiv:hep-ph/0508180.

[43] D. K. Hong, arXiv:hep-ph/0506097.

[44] M. Huang and I. Shovkovy, Nucl. Phys. A 729, 835 (2003) arXiv:hep-ph/0307273.

[45] M. Alford and Q. h. Wang, arXiv:hep-ph/0507269

[46] E. V. Gorbar, M. Hashimoto and V. A. Miransky, arXiv:hep-ph/0507303.

[47] A. Sedrakian, arXiv:nucl-th/0312053.

[48] D. T. Son and M. A. Stephanov, arXiv:cond-mat/0507586. 